\title{
THE QUEUE M|G|1 WITH MARKOV MODULATED ARRIVALS AND SERVICES*
}

\author{
G. J. K. REGTERSCHOT AND J. H. A. de SMIT
}

Twente University of Technology

\begin{abstract}
We study an $\mathrm{M}|\mathrm{G}| 1$ queue in which both the arrival rate and the service time distribution depend on the state of an underlying finite-state Markov chain. The solution is obtained by a matrix factorization method. This leads to results for waiting times and queue lengths both at arrival epochs and in continuous time. A numerical algorithm for the calculation of several quantities of interest is described and some numerical examples are given.
\end{abstract}

1. Introduction. We study an $M|G| 1$ queue with Markov modulated arrivals and services. Let $\left(X_{t}, t>0\right)$ be a Markov chain on $\{1,2, \ldots, N\}$ with indecomposable generator $Q$ with elements $Q_{i j}, i=1,2, \ldots, N ; j=1,2, \ldots, N$; and $q_{i}=-Q_{i i}, i=1$, $2, \ldots, N$. Denote its stationary distribution by $\pi=\left(\pi_{1}, \pi_{2}, \ldots, \pi_{N}\right)^{T}$, i.e., $Q^{T_{\pi}}=0$ and $1 \pi=1$, where 1 is the $N$-dimensional row vector with all components equal to 1 . Customers arrive at $T_{1}, T_{2}, \ldots ; T_{1}=0$. The service times are denoted by $S_{1}, S_{2}, \ldots$. If $X_{t}=i$ customers arrive according to a Poisson process with intensity $\lambda_{i}>0$ and an arriving customer has service time distribution $G_{i}$. Given $\left(X_{t}, t>0\right)$ the $S_{1}, S_{2}, \ldots$, are independent and also independent of the arrival process. Define $A_{n}=T_{n}-T_{n-1}$, $n=2,3, \ldots$; and $Y_{n}=X_{T_{n}}, n=1,2, \ldots$. Denote the waiting time of the $n$th customer by $W_{n}$ and assume that the system is initially empty, i.e. $W_{1}=0$. Moreover denote for $i=1,2, \ldots, N$;

$$
B_{i}(\phi)=\int_{0}^{\infty} \exp (-\phi x) d G_{i}(x) \text { and } \beta_{i}=\int_{0}^{\infty} x d G_{i}(x)=-B_{i}^{\prime}(0) .
$$

Throughout the paper we shall assume that there exists a $\delta>0$ such that the $B_{i}(\phi)$ can be continued analytically to the region $\operatorname{Re} \phi>-\delta$. The arrival process described above is called a Markov modulated arrival process. For this model we study the waiting times and queue lengths both at arrival epochs and in continuous time, generalizing known results for the ordinary $M|G| 1$ queue. A more general arrival process, the so-called N-process, has been studied by Ramaswami [4]. He analyses the $\mathrm{N}|\mathrm{G}| 1$ queue with a fixed service time distribution and obtains results for the busy period, the busy cycle, the steady-state queue length both at departure epochs and in continuous time, and for the virtual waiting time. Ramaswami claims that his results are computationally tractable, but he does not give any numerical examples. Burman and Smith [2] study the queue $M|G| 1$ with Markov modulated arrivals. They give approximations for the expected steady state waiting time and queue length both in light and in heavy traffic. The special case in which the arrival process is a switched Poisson process, i.e. $\left(X_{t}, t>0\right)$ is a 2-state Markov chain, has been analysed by van Hoorn and Seelen [5]. They obtain explicit numerical results for the steady-state queue length in continuous time.

Our model is a special case of the semi-Markov queue studied by Arjas [1]. The

\footnotetext{
'Received May 29, 1984; revised May 16, 1985.

AMS 1980 subject classification. Primary: 90B22; Secondary: 60K25.

IAOR 1973 subject classification. Main: Queues.

OR/MS Index 1978 subject classification. Primary: 695 Queues/Markovian.

Key words. M|G|l queue; Markov modulated arrivals and services; matrix factorization; actual waiting time; virtual waiting time; queue length.
} 
practical importance of the Markov modulated arrival process has been explained in [2], [4] and [5]. As we also allow the service time distribution to depend on the underlying Markov chain, the applicability of our model is even wider than that of the models discussed in the papers mentioned above.

The paper is organized as follows. In $\$ 2$ we derive a system of Wiener-Hopf-type equations, and in $\$ 3$ we give its solution using a factorization method. Results for the actual waiting time, the virtual waiting time and the queue length both at arrival epochs and in continuous time are given in \$4. Finally in $\$ 5$ a numerical algorithm is described and numerical examples are given. We shall use the following notations:

$\mathrm{I}(A)$ is the indicator function of the event $A ; x^{-}=\max (0, x) ; x^{-}=\min (0, x) ; M^{T}$ is the transpose of the matrix $M ; I$ is the $N \times N$-identity matrix.

2. Derivation of a system of Wiener-Hopf-type equations. For $j=1,2, \ldots, N$; $\operatorname{Re} \phi>0, \operatorname{Re} \eta>0$ and $|r|<1$ we define

$$
Z_{j}(r, \phi, \eta)=\sum_{n=1}^{\infty} r^{n} E\left(\exp \left(-\phi W_{n}-\eta T_{n}\right) 1\left(Y_{n}=j\right)\right)
$$

and we denote the $N$-dimensional column vector with components $Z_{j}(r, \phi, \eta)$ by $Z(r, \phi, \eta)$. In this section we derive a system of equations for the functions $Z_{j}(r, \phi, \eta)$, $j=1,2, \ldots, N$. This system turns out to be of Wiener-Hopf-type.

Let

$$
A_{i j}(\phi)=E\left(\exp \left(-\phi A_{n}\right) 1\left(Y_{n}=j\right) \mid Y_{n-1}=i\right)
$$

and $A(\phi)$ the $N \times N$-matrix with elements $A_{i j}(\phi), i=1,2, \ldots, N ; j=1,2, \ldots, N$. We prove the following lemma.

LEMma 2.1. For $\operatorname{Re} \phi>0$ the functions $A_{i j}$ satisfy the following system of equations

$$
\begin{gathered}
A_{i j}(\phi)=\frac{q_{i}+\lambda_{i}}{\phi+q_{i}+\lambda_{i}}\left[\frac{\lambda_{i}}{q_{i}+\lambda_{i}} \delta_{i j}+\sum_{\substack{k=1 \\
k \neq i}}^{N} \frac{Q_{i k}}{q_{i}+\lambda_{i}} A_{k j}(\phi)\right], \\
i=1,2, \ldots, N ; j=1,2, \ldots, N ;
\end{gathered}
$$

where $\delta_{i i}=1$ and $\delta_{i j}=0$ for $i \neq j$; or in matrix notation

$$
M(\phi) A(\phi)=\Lambda
$$

where $\Lambda=\operatorname{diag}\left(\lambda_{1}, \lambda_{2}, \ldots, \lambda_{N}\right)$ and $M(\phi)=\phi I+\Lambda-Q$.

Proor. Let $T_{n-1}+J_{n}$ be the first epoch after $T_{n-1}$ at which either a customer arrives or the Markov chain $\left(X_{t}, t>0\right)$ jumps to a new state. Then, given that $Y_{n-1}=i, J_{n}$ is exponentially distributed with parameter $q_{i}+\lambda_{i}$ and at $T_{n-1}+J_{n}$ with probability $\lambda_{i} /\left(q_{i}+\lambda_{i}\right)$ a customer arrives and with probability $Q_{i k} /\left(q_{i}+\lambda_{i}\right)$ the Markov chain $\left(X_{t}, t>0\right)$ jumps to state $k(k \neq i)$. By conditioning we thus obtain (2.1).

The process $\left(\left(W_{n}, T_{n}, Y_{n}\right), n=1,2, \ldots\right)$ is easily seen to be a Markov process, since $\left(\left(Y_{n}, T_{n}\right), n=1,2, \ldots\right)$ is a Markov renewal process. Denote $p_{j}=P\left(X_{0}=j\right), j=1$, $2, \ldots, N$; and let $p$ be the $N$-dimensional column vector with components $p_{j}$. Define for $j=1,2, \ldots, N, \operatorname{Re} \phi>0, \operatorname{Re} \eta>0,|r|<1$,

$$
V_{j}(r, \phi, \eta)=\sum_{n=1}^{\infty} r^{n+1} E\left(\left(1-\exp \left(\phi\left[W_{n}+S_{n}-A_{n+1}\right]^{-}\right)\right) \exp \left(-\eta T_{n+1}\right) 1\left(Y_{n+1}=j\right)\right),
$$

and let $V(r, \phi, \eta)$ be the $N$-dimensional column vector with components $V_{j}(r, \phi, \eta)$. The 
$N \times N$-matrix $H(r, \phi, \eta)$ is defined by

$$
H(r, \phi, \eta)=I-r A^{T}(\eta-\phi) B(\phi)
$$

where $B(\phi)=\operatorname{diag}\left(B_{1}(\phi), B_{2}(\phi), \ldots, B_{N}(\phi)\right)$.

Then we find the following system of Wiener-Hopf-type equations.

THEOREM 2.1 .

$$
H(r, \phi, \eta) Z(r, \phi, \eta)=r p+V(r,-\phi, \eta)
$$

for $\operatorname{Re} \phi=0, \operatorname{Re} \eta>0$ and $|r|<1$.

Proof. Using the identity

$$
\exp \left(-\phi x^{+}\right)+\exp \left(-\phi x^{-}\right)=\exp (-\phi x)+1,
$$

we have for $\operatorname{Re} \phi=0, \operatorname{Re} \eta \geqslant 0,|r|<1$,

$$
\begin{gathered}
E\left(\exp \left(-\phi W_{n+1}-\eta T_{n+1}\right) 1\left(Y_{n+1}=j\right)\right) \\
=E\left(\exp \left(-\phi\left[W_{n}+S_{n}-A_{n+1}\right]^{+}-\eta T_{n+1}\right) 1\left(Y_{n+1}=j\right)\right) \\
=E\left(\exp \left(-\phi\left(W_{n}+S_{n}-A_{n+1}\right)-\eta T_{n+1}\right) 1\left(Y_{n+1}=j\right)\right) \\
+E\left(\left(1-\exp \left(-\phi\left[W_{n}+S_{n}-A_{n+1}\right]^{-}\right)\right) \exp \left(-\eta T_{n+1}\right) 1\left(Y_{n+1}=j\right)\right) \text { and } \\
E\left(\exp \left(-\phi\left(W_{n}+S_{n}-A_{n+1}\right)-\eta T_{n+1}\right) 1\left(Y_{n+1}=j\right)\right) \\
=\sum_{i=1}^{N} E\left(\exp \left(-\phi W_{n}-\eta T_{n}\right) 1\left(Y_{n}=i\right)\right) \\
\cdot E\left(\exp \left(-\phi S_{n}-(\eta-\phi) A_{n+1}\right) 1\left(Y_{n+1}=j\right) \mid Y_{n}=i\right) \\
=\sum_{i=1}^{N} E\left(\exp \left(-\phi W_{n}-\eta T_{n}\right) 1\left(Y_{n}=i\right)\right) B_{i}(\phi) A_{i j}(\eta-\phi),
\end{gathered}
$$

so for $\operatorname{Re} \phi=0$,

$$
Z_{j}(r, \phi, \eta)=r P\left(X_{0}=j\right)+r \sum_{i=1}^{N} Z_{i}(r, \phi, \eta) B_{i}(\phi) A_{i j}(\eta-\phi)+V_{j}(r,-\phi, \eta),
$$

which proves the theorem.

3. Solution of the system of Wiener-Hopf-type equations. We solve the system (2.3) by a factorization method which is similar to the method applied in [3]. First we prove an auxiliary lemma. Let $\rho=\sum_{i=1}^{N} \pi_{i} \lambda_{i} \beta_{i}$ and $\lambda=\sum_{i=1}^{N} \pi_{i} \lambda_{i}$, and define

$$
L(r, \phi, \eta)=(\eta-\phi) I+(I-r B(\phi)) \Lambda-Q^{T} \text {. }
$$

LEMMA 3.1. The $N$ eigenvalues of $\Lambda-Q^{T}$ all lie in the right half-plane $\operatorname{Re} \phi>0$; for $|r|<1, \operatorname{Re} \eta>0$ or $|r|<1, \operatorname{Re} \eta>0$, det $L(r, \phi, \eta)$ has exactly $N$ zeros in the right half-plane $\operatorname{Re} \phi>0$.

If $\rho<1$, det $L(1, \phi, 0)$ has exactly $N-1$ zeros in the right half-plane $\operatorname{Re} \phi>0$ and $a$ simple zero at 0 .

Proof. For $d>0$ let $C_{d}$ be the closed contour consisting of the part of the imaginary axis running from - id to $i d$ and the half-circle with centre at the origin and radius $d$ running clockwise from id to $-i d$.

Let $d_{0}=|\eta|+2 \max _{1<i<N}\left(\lambda_{i}+q_{i}\right)$. Then on $C_{d}$ with $d>d_{0}$ we have for $|r|<1$, 
$\operatorname{Re} \eta>0$, or $|r|<1, \operatorname{Re} \eta>0$,

$$
\left|\eta-\phi+q_{i}+\lambda_{i}\right|>q_{i}+\lambda_{i}\left|r B_{i}(\phi)\right|, \quad i=1,2, \ldots, N .
$$

From Theorem 1 of Appendix 2 of [3] it follows that

$$
\operatorname{det}\left[(\eta-\phi) I+(I-r B(\phi)) \Lambda-Q^{T}\right]
$$

has the same number of zeros (counted according to their order) inside $C_{d}$ as

$$
\operatorname{det}\left[(\eta-\phi) I+\operatorname{diag}\left(q_{1}+\lambda_{1}, q_{2}+\lambda_{2}, \ldots, q_{N}+\lambda_{N}\right)\right]=\prod_{i=1}^{N}\left(\eta-\phi+q_{i}+\lambda_{i}\right) .
$$

This proves the first part of the lemma. Moreover we see that $\operatorname{det} M(0) \neq 0$ and $\operatorname{det} L(r, 0, \eta) \neq 0$ for $|r|<1, \operatorname{Re} \eta>0$, or $|r|<1, \operatorname{Re} \eta>0$. To prove the second part consider the matrix

$$
L(r, \phi, 0)=-\phi I+(I-r B(\phi)) \Lambda-Q^{T} .
$$

The matrix $L^{*}(r, \phi)$ is obtained from $L(r, \phi, 0)$ by adding all rows to the first row so that

$$
L_{i j}^{*}(r, \phi)=-\phi+\left(1-r B_{j}(\phi)\right) \lambda_{j}, \quad j=1,2, \ldots, N .
$$

Let $\mu(r)$ be the function defined by

$$
\operatorname{det} L(r, \mu(r), 0)=\operatorname{det} L^{*}(r, \mu(r))=0
$$

and $\mu(1)=0$. Using the implicit function theorem we shall prove that there is a neighbourhood of 1 in which the function $\mu(r)$ is uniquely defined and continuously differentiable. Since we have assumed that there exists a $\delta>0$ such that the $B_{i}(\phi)$ can be continued analytically to the region $\operatorname{Re} \phi>-\delta$, there exists in $R \times C$ a neighbourhood of $(1,0)$ in which det $L^{*}(r, \phi)$ is continuously differentiable with respect to both $r$ and $\phi$. Moreover $\operatorname{det} L^{*}(1,0)=0$. For small $\phi$ we have

$$
L_{i j}^{*}(1, \phi)=-\phi\left(1-\beta_{j} \lambda_{j}\right)+o(\phi), \quad \phi \rightarrow 0
$$

so that

$$
\begin{aligned}
\operatorname{det} L^{*}(1, \phi)=-\phi \operatorname{det} L^{*}+o(\phi), \quad \phi \rightarrow 0, & \text { where } \\
L_{1 j}^{*} & =1-\beta_{j} \lambda_{j}, \quad j=1,2, \ldots, N ; \\
L_{i j}^{*} & =-Q_{j i}, \quad i=2,3, \ldots, N ; j=1,2, \ldots, N .
\end{aligned}
$$

Consequently

$$
\left.\frac{\partial}{\partial \phi} \operatorname{det} L^{*}(1, \phi)\right|_{\phi=0}=-\operatorname{det} L^{*}
$$

We immediately see that

$$
L^{*} \pi=(1-\rho) e_{1}
$$

where $e_{1}=(1,0, \ldots, 0)^{T}$. Since $Q^{T} \pi=0$ and $Q^{T}$ has rank $N-1, \pi$ is the only solution of (3.5) and hence $\operatorname{det} L^{*} \neq 0$. We see that all conditions of the implicit function theorem are satisfied so that in a neighbourhood of 1 the function $\mu(r)$ is uniquely defined and continuously differentiable. We consider this function for $r$ close to 1 , so

$$
\begin{gathered}
\mu(r)=-(1-r) \mu^{\prime}(1)+o(1-r), \quad r \rightarrow 1, \text { and } \\
L_{i j}^{*}(r, \mu(r))=(1-r)\left[\mu^{\prime}(1)+\left(1-\beta_{j} \mu^{\prime}(1)\right) \lambda_{j}\right]+o(1-r), \quad r \rightarrow 1 .
\end{gathered}
$$


Since $\operatorname{det} L^{*}(r, \mu(r))=0$,

$$
\begin{aligned}
0 & =\left.\frac{d}{d r} \operatorname{det} L^{*}(r, \mu(r))\right|_{r=1}=-\operatorname{det} L^{\circ}, \quad \text { where } \\
L^{\circ}{ }_{1 j} & =\mu^{\prime}(1)+\left(1-\beta_{j} \mu^{\prime}(1)\right) \lambda_{j}, \quad j=1,2, \ldots, N ; \quad \text { and } \\
L_{i j}^{\circ} & =-Q_{j i}, \quad i=2,3, \ldots, N ; j=1,2, \ldots, N .
\end{aligned}
$$

Now we must have $L^{\circ} \pi=0$ (otherwise $\operatorname{det} L^{\circ}$ would not equal 0 ) and in particular $\sum_{j=1}^{N} L_{1 j}^{\circ} \pi_{j}=0$, or with $(3.6) \mu^{\prime}(1)=-\lambda /(1-\rho)$, so that

$$
\mu(r)=(1-r) \lambda /(1-\rho)+o(1-r), \quad r \rightarrow 1 .
$$

The inequalities $\left|-\phi+\lambda_{i}+q_{i}\right|>q_{i}+\lambda_{i}\left|r B_{i}(\phi)\right|, i=1,2, \ldots, N$, hold for $r<1, \operatorname{Re} \phi$ $=0$, and for $r=1, \operatorname{Re} \phi=0, \phi \neq 0$. This implies (see Lemma 1 of Appendix 2 of [3]) that, for $r<1$, det $H(r, \phi, 0)$ has no zeros on the imaginary axis $\operatorname{Re} \phi=0$, while for $r=1$ it can have no zeros on the imaginary axis except at $\mathbf{0}$. According to the first part of the present theorem there are for $r<1$ exactly $N$ zeros in the right half-plane $\operatorname{Re} \phi>0$. These zeros are continuous functions of $r$. From (3.7) we see that for $\rho<1$ and $r<1$ and sufficiently close to 1 the function $\mu(r)$ is one of these zeros. Since $\operatorname{det} H(1, \phi, 0)$ has a simple zero at 0 and no zeros elsewhere on the imaginary axis we see that for $r \uparrow 1$ the zero $\mu(r)$ is the only zero which tends to the imaginary axis while the other zeros remain in the right half-plane $\operatorname{Re} \phi>0$. This proves the second part of the theorem.

From (2.2) we have $A(\phi)=M^{-1}(\phi) \Lambda$ so that

$$
H(r, \phi, \eta)=I-r \Lambda\left(M^{r}(\eta-\phi)\right)^{-1} B(\phi) .
$$

Consequently

$$
\begin{gathered}
L(r, \phi, \eta)=M^{T}(\eta-\phi) \Lambda^{-1} H(r, \phi, \eta) \Lambda \text { and } \\
\operatorname{det} L(r, \phi, \eta)=\operatorname{det} M(\eta-\phi) \operatorname{det} H(r, \phi, \eta)
\end{gathered}
$$

Let $\mu_{1}(r, \eta), \ldots, \mu_{N}(r, \eta)$ be the $N$ zeros in the right half-plane of $\operatorname{det} L(r, \phi, \eta)$, with $\mu_{1}(1,0)=0$, and let $\nu_{1}, \ldots, \nu_{N}$ be the $N$ eigenvalues of $\Lambda-Q^{T}$. Note that for $\operatorname{Re} \eta>0, \mu_{i}(0, \eta)=\nu_{i}+\eta, i=1,2, \ldots, N$. We next impose the following condition.

Condition 3.1. $\mu_{1}(r, \eta), \ldots, \mu_{N}(r, \eta)$ and $\nu_{1}+\eta, \ldots, \nu_{N}+\eta$ are all distinct.

As in [3] we see that this condition will always be satisfied if $\nu_{1}, \nu_{2}, \ldots, \nu_{N}$ are distinct, except possibly for countably many values of $\eta$ if $r$ is fixed or countably many values of $r$ if $\eta$ is fixed. Hence from (3.9) it follows that $\mu_{1}(r, \eta), \ldots, \mu_{N}(r, \eta)$ are the zeros in the right half-plane $\operatorname{Re} \phi>0$ of $\operatorname{det} H(r, \phi, \eta)$.

For $i=1,2, \ldots, N$, let $D_{i}$ be a (nonunique) nonzero row vector satisfying

$$
D_{i} H\left(r, \mu_{i}(r, \eta), \eta\right)=0 \text {, }
$$

and let $D$ be the $N \times N$-matrix the $i$ th row of which is $D_{i}$. For $i=1,2, \ldots, N$, let $R^{i}$ be a right eigenvector of $\Lambda-Q^{T}$ corresponding to the eigenvalue $\nu_{i}$, while $R$ is the matrix the $i$ th column of which is $R^{i}$. Then

$$
\Lambda-Q^{r}=R \operatorname{diag}\left(\nu_{1}, \nu_{2}, \ldots, \nu_{N}\right) R^{-1} .
$$

Define the $N \times N$-matrix $S$ by

$$
S_{i j}=\frac{1}{\nu_{j}+\eta-\mu_{i}(r, \eta)} D_{i} \Lambda R^{j}
$$

Note that $D, R$ and $S$ depend on $r$ and $\eta$. We assume that the following condition holds. 
Condition 3.2. $\operatorname{det} S \neq 0$.

Again we note that this condition will always be satisfied except possibly for countably many values of $\eta$ if $r$ is fixed or countably many values of $r$ if $\eta$ is fixed.

Define

$$
\begin{gathered}
K(r, \phi, \eta)=I-C J(r, \phi, \eta) D, \quad \text { where } \\
C=\Lambda R S^{-1} \text { and } J(r, \phi, \eta)=\operatorname{diag}\left(\frac{1}{\phi-\mu_{1}(r, \eta)}, \ldots, \frac{1}{\phi-\mu_{N}(r, \eta)}\right) .
\end{gathered}
$$

We can now state and prove the factorization theorem.

THEOREM 3.1. If Conditions 3.1 and 3.2 hold then

$$
\operatorname{det} K(r, \phi, \eta)=\prod_{i=1}^{N}\left[\frac{\phi-\nu_{i}-\eta}{\phi-\mu_{i}(r, \eta)}\right],
$$

(ii) for $\operatorname{Re} \phi=0$,

$$
H(r, \phi, \eta)=H^{-}(r, \phi, \eta) H^{+}(r, \phi, \eta), \quad \text { where }
$$

(a) $H^{-}(r, \phi, \eta)=K^{-1}(r, \phi, \eta)$ is analytic for $\operatorname{Re} \phi<0$ and bounded and continuous for $\operatorname{Re} \phi<0$

(b) $H^{+}(r, \phi, \eta)=K(r, \phi, \eta) H(r, \phi, \eta)$ is analytic for $\operatorname{Re} \phi>0$ and bounded and continuous for $\operatorname{Re} \phi>0$.

Proof. From (3.13) we see that $\mu_{i}(r, \eta)$ is either a simple pole of $\operatorname{det} K(r, \phi, \eta)$ or det $K(r, \phi, \eta)$ is analytic at $\mu_{i}(r, \eta)$. Since $\lim _{|\phi| \rightarrow \infty} K(r, \phi, \eta)=I$ we have

$$
\operatorname{det} K(r, \phi, \eta)=g(r, \phi, \eta) / \prod_{i=1}^{N}\left(\phi-\mu_{i}(r, \eta)\right) \text {, }
$$

where $g(r, \phi, \eta)$ is a polynomial of $\phi$ of degree $N$. From (3.9) and Condition 3.1 it follows that det $H(r, \phi, \eta)$ is of the form

$$
\operatorname{det} H(r, \phi, \eta)=f(r, \phi, \eta) \prod_{i=1}^{N}\left[\frac{\phi-\mu_{i}(r, \eta)}{\phi-\eta-\nu_{i}}\right],
$$

where $f(r, \phi, \eta)$ is bounded away from 0 for $\operatorname{Re} \phi>0$.

Expression (3.11) implies

$$
\left[M^{T}(\eta-\phi)\right]^{-1}=R \operatorname{diag}\left(\frac{1}{\nu_{1}+\eta-\phi}, \ldots, \frac{1}{\nu_{N}+\eta-\phi}\right) R^{-1},
$$

so that from (3.8)

$$
\begin{aligned}
H^{+}(r, \phi, \eta) & =K(r, \phi, \eta) H(r, \phi, \eta) \\
& =K(r, \phi, \eta)+r \sum_{i=1}^{N} \frac{1}{\phi-\eta-\nu_{i}} K(r, \phi, \eta) \Lambda R^{i} R_{i}^{-1} B(\phi)
\end{aligned}
$$

where $R_{i}^{-1}$ is the $i$ th row of $R^{-1}$. Note that $R_{i}^{-1}$ is a left eigenvector of $\Lambda-Q^{T}$ corresponding to the eigenvalue $\nu_{i}$. Moreover from (3.13) we have

$$
\begin{aligned}
K\left(r, p_{i}+\eta, \eta\right) \Lambda R^{i} & =\Lambda R^{i}-\sum_{j=1}^{N} C^{j} \frac{1}{\eta_{i}+\eta-\mu_{j}(r, \eta)} D_{j} \Lambda R^{i} \\
& =\Lambda R^{i}-\sum_{j=1}^{N} C^{j} S_{j i}=0
\end{aligned}
$$


where $C^{j}$ is the $j$ th column of $C$. Now (3.17) yields

$$
\lim _{\phi \rightarrow \nu_{i}+\eta}\left(\phi-\nu_{i}-\eta\right) H^{+}(r, \phi, \eta)=r K\left(r, \nu_{i}+\eta, \eta\right) \Lambda R^{i} R_{i}^{-1} B\left(\nu_{i}+\eta\right)=0 .
$$

We see that $H^{+}(r, \phi, \eta)$ has no poles at $\nu_{i}+\eta, i=1,2, \ldots, N$; but from (3.14) and (3.15)

$$
\operatorname{det} H^{+}(r, \phi, \eta)=f(r, \phi, \eta) g(r, \phi, \eta) / \prod_{i=1}^{N}\left(\phi-\nu_{i}-\eta\right),
$$

and hence

$$
g(r, \phi, \eta)=\prod_{i=1}^{N}\left(\phi-\nu_{i}-\eta\right)
$$

which proves part (i) of the theorem.

With (3.13) we have

$$
H^{+}(r, \phi, \eta)=H(r, \phi, \eta)-C \operatorname{diag}\left(\frac{1}{\phi-\mu_{1}(r, \eta)}, \ldots, \frac{1}{\phi-\mu_{N}(r, \eta)}\right) D H(r, \phi, \eta)
$$

so that

$$
\lim _{\phi \rightarrow \mu_{i}(r, \eta)}\left(\phi-\mu_{i}(r, \eta)\right) H^{+}(r, \phi, \eta)=-C^{i} D_{i} H\left(r, \mu_{i}(r, \eta), \eta\right)=0,
$$

and the proof of part (ii) is complete.

Remark. The Wiener-Hopf factors $H^{-}$and $H^{+}$satisfying

$$
H(r, \phi, \eta)=H^{-}(r, \phi, \eta) H^{+}(r, \phi, \eta)
$$

are unique up to multiplication by a constant matrix. This means that our factorization is equivalent to the one given by Arjas [1, Lemma 2.2]. In fact the factorizations are identical since in both cases $\lim _{\phi \rightarrow-\infty} H^{-}(r, \phi, \eta)=I$. Arjas gives a probabilistic interpretation to the factors but his method does not lead to explicit computationally tractable results. Denote $U_{n}=S_{1}+\cdots+S_{n}, n=1,2, \ldots ;$ and $M=\inf \left\{n>0 \mid T_{n+1}\right.$ $\left.>U_{n}\right\}$, then $M$ is the number of customers during the first busy period, $U_{M}$ the length of the first busy period and $T_{M+1}$ the length of the first busy cycle. From Arjas [1] we now have the following probabilistic interpretation of our Wiener-Hopf factors

$$
\begin{aligned}
& H_{j i}^{-}(r, \phi, \eta)=\delta_{i j}-E\left(r^{M} \exp \left(-\phi\left(U_{M}-T_{M+1}\right)-\eta T_{M+1}\right) 1\left(Y_{M+1}=j\right) \mid Y_{1}=i\right) \text { and } \\
& \quad\left[H^{+}(r, \phi, \eta)\right]_{j i}^{-1} \\
& \quad=\delta_{i j}+\sum_{n=1}^{\infty} r^{n} E\left(\exp \left(-\phi\left(U_{n}-T_{n+1}\right)-\eta T_{n+1}\right) 1\left(M>n, Y_{n+1}=j\right) \mid Y_{1}=i\right) .
\end{aligned}
$$

We can now give the solution of the equations (2.3).

Throrem 3.2. If Conditions 3.1 and 3.2 hold then for $\operatorname{Re} \phi>0$,

$$
K(r, \phi, \eta) H(r, \phi, \eta) Z(r, \phi, \eta)=r K(r, 0, \eta) p
$$

if $|r|<1, \operatorname{Re} \eta>0$, or $|r|<1, \operatorname{Re} \eta>0$.

PRoof. With a standard Wiener-Hopf argument (see [3]) we have for $\operatorname{Re} \phi>0$,

$$
H^{+}(r, \phi, \eta) Z(r, \phi, \eta)=H^{+}(r, 0, \eta) Z(r, 0, \eta) .
$$

Moreover from (2.3)

$$
H(r, 0, \eta) Z(r, 0, \eta)=r p
$$

so that the theorem is proved. 


\section{Waiting time and number of customens in the system.}

4.1. Actual waiting time. The process $\left(\left(W_{n}, Y_{n}\right), n=1,2, \ldots\right)$ is regenerative where for any $i=1,2, \ldots, N$; the state $(0, i)$ can be chosen to be the regenerative state. We easily see that the return times are aperiodic, so that

$$
\lim _{n \rightarrow \infty} P\left(W_{n} \leqslant x, Y_{n}=i\right)
$$

for $x>0$ exists. If this limit is 0 no limiting distribution exists, if it is positive $\left(W_{n}, Y_{n}\right)$ converges weakly to a stationary random vector $(W, Y)$. From (3.13) we see that

$$
\lim _{\uparrow 1}(1-r) K(r, 0,0) p=C^{1} \lim _{r \uparrow 1} \frac{1-r}{\mu_{1}(r, 0)} D_{1} p,
$$

where $C^{1}$ is the first column of $C$. Since $H(1,0,0)=I-A^{T}(0)$, where $A(0)$ is the transition matrix of the Markov chain $\left(Y_{n}, n=1,2, \ldots\right)$, we see from (3.10) that $D_{1}=D_{11}(1,1, \ldots, 1)$, so that $D_{1} p=D_{11}$. From the proof of Lemma 3.1 we have

$$
\begin{aligned}
\lim _{\uparrow 1} \frac{1-r}{\mu_{1}(r, 0)} & =(1-\rho) / \lambda, & & \text { for } \rho<1, \\
& =0, & & \text { for } \rho>1 .
\end{aligned}
$$

Part (i) of Theorem 3.1 implies that $C_{i 1} \neq 0$ for at least one $i$, if Conditions 3.1 and 3.2 hold for $r=1$ and $\eta=0$.

Denote

$$
\begin{gathered}
\mu_{i}=\mu_{i}(1,0), \quad i=1,2, \ldots, N ; \text { where } \mu_{1}=0 ; \\
H(\phi)=H(1, \phi, 0) ; \\
\tilde{K}(\phi)=I-C \operatorname{diag}\left(0, \frac{1}{\phi-\mu_{2}}, \frac{1}{\phi-\mu_{3}}, \ldots, \frac{1}{\phi-\mu_{N}}\right) D ; \\
H^{+}(\phi)=\tilde{K}(\phi) H(\phi)-\frac{1}{\phi} C^{1} D_{1} H(\phi), \quad \phi \neq 0, \\
=\tilde{K}(0) H(0)-C^{1} D_{1} H^{\prime}(0), \quad \phi=0 ;
\end{gathered}
$$

and let $Z(\phi)$ be the $N$-dimensional column vector with components

$$
Z_{j}(\phi)=E(\exp (-\phi W) 1(Y=j)), \quad j=1,2, \ldots, N ;
$$

then from Theorem 3.2 we have for $\operatorname{Re} \phi>0$,

$$
\begin{aligned}
H^{+}(\phi) Z(\phi) & =C^{1} D_{11}(1-\rho) / \lambda & & \text { for } \rho<1, \\
& =0 & & \text { for } \rho>1 .
\end{aligned}
$$

Let $Z_{0}$ be the column vector with components $Z_{j}=P(W=0, Y=j)=\lim _{\phi \rightarrow \infty} Z_{j}(\phi)$. It is easily seen that if $Z_{j}>0$ for some $j$, then $Z_{j}>0$ for all $j=1,2, \ldots, N$; and consequently

$$
\begin{aligned}
Z_{0} & =C^{1} D_{11}(1-\rho) / \lambda & & \text { for } \rho<1, \\
& =0 & & \text { for } \rho>1 ;
\end{aligned}
$$

where all components of $C^{1} D_{11}$ are strictly positive. The moments $E\left(W^{k} 1(Y=j)\right)$, $k=1,2, \ldots$, are obtained by differentiating (4.3) setting $\phi=0$ and solving the resulting linear equations. In order to obtain the first two moments we solve $Z(0)$, 
$Z^{\prime}(0)$ and $Z^{\prime \prime}(0)$ from the equations

$$
\begin{gathered}
H^{+}(0) Z(0)=Z_{0}, \quad\left(H^{+}(0)\right)^{\prime} Z(0)+H^{+}(0) Z^{\prime}(0)=0, \quad \text { and } \\
\left(H^{+}(0)\right)^{\prime \prime} Z(0)+2\left(H^{+}(0)\right)^{\prime} Z^{\prime}(0)+H^{+}(0) Z^{\prime \prime}(0)=0, \quad \text { where } \\
H^{+}(0)=\tilde{K}(0) H(0)-C^{1} D_{1} H^{\prime}(0), \\
\left(H^{+}(0)\right)^{\prime}=\tilde{K}^{\prime}(0) H(0)+\tilde{K}(0) H^{\prime}(0)-\frac{1}{2} C^{1} D_{1} H^{\prime \prime}(0) \quad \text { and } \\
\left(H^{+}(0)\right)^{\prime \prime}=\tilde{K}^{\prime \prime}(0) H(0)+2 \tilde{K}^{\prime}(0) H^{\prime}(0)+\tilde{K}(0) H^{\prime \prime}(0)-\frac{1}{3} C^{1} D_{1} H^{\prime \prime \prime}(0) .
\end{gathered}
$$

Then

$$
\begin{array}{cc}
E(W 1(Y=j))=-Z_{j}^{\prime}(0), & j=1,2, \ldots, N \\
E\left(W^{2} 1(Y=j)\right)=Z_{j}^{\prime \prime}(0), & j=1,2, \ldots, N
\end{array}
$$

4.2. Virtual waiting time. Let $W_{t}^{*}$ be the virtual waiting time at time $t$, then

$$
W_{t}^{*}=\left[W_{N_{t}}+S_{N_{t}}+T_{N_{t}}-t\right]^{+} \text {, }
$$

where $N_{t}$ is the number of customers arriving during $[0, t]$. Consequently for $\operatorname{Re} \phi>0$,

$$
\begin{aligned}
& E\left(\exp \left(-\phi W_{t}^{*}\right) 1\left(X_{t}=k\right)\right) \\
&=\sum_{n=1}^{\infty} E\left(\exp \left(-\phi\left[W_{n}+S_{n}+T_{n}-t\right]^{+}\right) 1\left(T_{n} \leqslant t<T_{n+1}, X_{t}=k\right)\right) \\
&=\sum_{n=1}^{\infty} \sum_{l=1}^{N} \int_{0}^{t} P\left(A_{2}>t-u, X_{t-u}=k \mid X_{0}=l\right) \\
& \quad \cdot d_{u} E\left(\exp \left(-\phi\left[W_{n}+S_{n}+T_{n}-t\right]^{+}\right) 1\left(Y_{n}=l, T_{n} \leqslant u\right)\right) .
\end{aligned}
$$

Let $P(t)$ be the transition matrix of the Markov chain $\left(X_{t}, t \geqslant 0\right)$ and

$$
\tilde{P}(\phi)=\int_{0}^{\infty} \exp (-\phi t) P(t) d t, \quad \operatorname{Re} \phi>0,
$$

then

$$
\tilde{P}(\phi)=(\phi I-Q)^{-1}
$$

Since

$$
P\left(A_{2}>t, X_{t}=k \mid X_{0}=l\right)=P_{l k}(t)-\sum_{m=1}^{N} \int_{0}^{t} P_{m k}(t-u) d P\left(A_{2} \leqslant u, Y_{1}=m \mid Y_{0}=l\right)
$$

we have from (2.2) and (4.9)

$$
\begin{aligned}
& \int_{0}^{\infty} \exp (-\eta t) P\left(A_{2}>t, X_{t}=k \mid X_{0}=l\right) d t \\
& \quad=\tilde{P}_{l k}(\eta)-\sum_{m=1}^{N} \tilde{P}_{m k}(\eta) A_{l m}(\eta)=\left[M^{-1}(\eta)\right]_{l k} .
\end{aligned}
$$

From the identity

$$
\exp \left(-\phi x^{+}\right)=\frac{1}{2 \pi i} \int_{-i \infty+0}^{i \infty 0+0} \frac{d \xi}{\xi} \frac{\phi}{\phi-\xi} \exp (-\xi x), \quad \operatorname{Re} \phi>\operatorname{Re} \xi>0,
$$


where the path of integration is a line parallel to the imaginary axis, we have

$$
\begin{aligned}
& E\left(\exp \left(-\phi\left[W_{n}+S_{n}+T_{n}-t\right]^{+}\right) 1\left(Y_{n}=l, T_{n}<u\right)\right) \\
& \quad=\frac{1}{2 \pi i} \int_{-i \infty 0+0}^{i \infty 0+0} \frac{d \xi}{\xi} \frac{\phi}{\phi-\xi} \exp (\xi t) B_{l}(\xi) E\left(\exp \left(-\xi\left(W_{n}+T_{n}\right)\right) 1\left(Y_{n}=l, T_{n}<u\right)\right) .
\end{aligned}
$$

Combining (4.8), (4.10) and (4.12) yields

$$
\begin{aligned}
\int_{0}^{\infty} \exp (-\eta t) E\left(\exp \left(-\phi W_{t}^{*}\right) 1\left(X_{t}=k\right)\right) d t \\
=\sum_{n=1}^{\infty} \sum_{l=1}^{N} \frac{1}{2 \pi i} \int_{-i \infty+0}^{i \infty+0} \frac{d \xi}{\xi} \frac{\phi}{\phi-\xi} M^{-1}(\eta-\xi)_{l k} B_{l}(\xi) \\
\quad \cdot E\left(\exp \left(-\xi W_{n}-\eta T_{n}\right) 1\left(Y_{n}=l\right)\right) .
\end{aligned}
$$

If $Z^{*}(\eta, \phi)$ is the column vector with components

$$
Z_{k}^{*}(\eta, \phi)=\int_{0}^{\infty} \exp (-\eta t) E\left(\exp \left(-\phi W_{i}^{*}\right) 1\left(X_{t}=k\right)\right) d t
$$

then (4.13) becomes

$$
Z^{*}(\eta, \phi)=\frac{1}{2 \pi i} \int_{-i \infty+0}^{i \infty+0} \frac{d \xi}{\xi} \frac{\phi}{\phi-\xi}\left[M^{T}(\eta-\xi)\right]^{-1} B(\xi) Z(1, \xi, \eta),
$$

$\operatorname{Re} \phi>\operatorname{Re} \xi>0, \operatorname{Re} \eta>0$.

The interchanges of the order of integration and summation which were made in the above derivation are justified by absolute convergence and Fubini's theorem. From Theorem 3.2 we have

$$
\Lambda\left[M^{T}(\eta-\phi)\right]^{-1} B(\phi) Z(1, \phi, \eta)=Z(1, \phi, \eta)-K^{-1}(1, \phi, \eta) K(1,0, \eta) p .
$$

Inserting this into (4.14) and applying contour integration gives for $\operatorname{Re} \eta>0, \operatorname{Re} \phi \geqslant 0$,

$$
Z^{*}(\eta, \phi)=\Lambda^{-1} Z(1, \phi, \eta)-\Lambda^{-1} p
$$

The process $\left(\left(W_{t}^{*}, X_{t}\right), t>0\right)$ is regenerative, where the regeneration points are the epochs at which the process enters a state $(0, i)$ for some $i, 1<i<N$. Since the times between regeneration points are nonarithmetic $\lim _{t \rightarrow \infty} E\left(\exp \left(-\phi W_{t}^{*}\right) 1\left(X_{t}=i\right)\right)$ exists. Denote this limit by $Z_{i}^{*}(\phi)$ and let $Z^{*}(\phi)$ be the $N$-dimensional column vector with components $Z_{i}^{*}(\phi)$. Similar to (4.2) we have

$$
\begin{aligned}
\lim _{\eta \downarrow 0} \frac{\eta}{\mu_{1}(1, \eta)} & =1-\rho & & \text { for } \rho<1, \\
& =0 & & \text { for } \rho>1,
\end{aligned}
$$

so that

$$
\begin{aligned}
\lim _{\eta \downarrow 0} \eta K(1,0, \eta) p & =C^{1} D_{11}(1-\rho) & & \text { for } \rho<1, \\
& =0 & & \text { for } \rho>1 .
\end{aligned}
$$

From Theorem 3.2 and (4.3) it therefore follows that

$$
\lim _{\eta 0} \eta Z(1, \phi, \eta)=\lambda Z(\phi)
$$

We see that $\left(W_{i}^{*}, X_{t}\right)$ converges weakly to a random vector $\left(W^{*}, X\right)$ iff $\rho<1$, and 
from (4.15) we have for $\operatorname{Re} \phi>0$,

$$
Z^{*}(\phi)=\lambda \Lambda^{-1} Z(\phi)
$$

Let $Z_{0}^{*}$ be the column vector with components $Z_{j}^{*}=P\left(W^{*}=0, X=j\right)$, then

$$
Z_{0}^{*}=\lambda \Lambda^{-1} Z_{0}=\Lambda^{-1} C^{1} D_{11}(1-\rho),
$$

while with (4.17) the moments of $W^{*}$ are easily expressed in those of $W$.

4.3. Number of customers in the system at arrival epochs. Let $C_{n}$ be the number of customers in the system at $T_{n}-$, i.e. just before the arrival of the $n$th customer. Since

$$
\begin{gathered}
\left\{C_{n}<j\right\}=\Omega, \quad n=1,2, \ldots, j+1 ; \\
\left\{C_{n+j+1}<j\right\}=\left\{T_{n}+W_{n}+S_{n}<T_{n+j+1}\right\}, \quad n=1,2, \ldots ;
\end{gathered}
$$

we find by straightforward calculations

$$
\begin{aligned}
\sum_{n=1}^{\infty} r^{n} E\left(s c_{n} 1\left(Y_{n}=k\right)\right)= & r \sum_{i=1}^{N} p_{l}[I-r s A(0)]_{l k}^{-1}+r(1-s) \sum_{i=1}^{N} \sum_{j=0}^{\infty}(r s)^{j} \\
& \times \sum_{n=1}^{\infty} r^{n} P\left(T_{n}+W_{n}+S_{n}<T_{n+j+1}, Y_{n}=i, Y_{n+j+1}=k\right) .
\end{aligned}
$$

We use the identity

$$
1(x<0)+\frac{1}{2} 1(x=0)=\frac{1}{2 \pi i} \int_{-i \infty+0+0}^{i \infty+0} \frac{d \xi}{\xi} \exp (-\xi x)
$$

where $\int_{-i \infty+0}^{i \infty 0+0}$ stands for $\lim _{R \rightarrow \infty} \int_{-i R+\delta}^{i R+\delta}$ with $\delta>0$ and sufficiently small. Then we have

$$
\begin{aligned}
P\left(T_{n}\right. & \left.+W_{n}+S_{n}<T_{n+j+1}, Y_{n}=i, Y_{n+j+1}=k\right) \\
& =\frac{1}{2 \pi i} \int_{-i \infty+0}^{i \infty+0} \frac{d \xi}{\xi} E\left(\exp \left(-\xi\left(W_{n}+S_{n}+T_{n}-T_{n+j+1}\right)\right) 1\left(Y_{n}=i, Y_{n+j+1}=k\right)\right) \\
& =\frac{1}{2 \pi i} \int_{-i \infty+0}^{i \infty+0} \frac{d \xi}{\xi} E\left(\exp \left(-\xi W_{n}\right) 1\left(Y_{n}=i\right)\right) B_{i}(\xi)\left[A^{j+1}(-\xi)\right]_{i k} .
\end{aligned}
$$

Inserting this into (4.20) yields

$$
\begin{aligned}
\sum_{n=1}^{\infty} r^{n} E\left(s c_{n} 1\left(Y_{n}=k\right)\right)= & r \sum_{l=1}^{N} p_{l}[I-r s A(0)]_{i k}^{-1} \\
& +r(1-s) \sum_{i=1}^{N} \frac{1}{2 \pi i} \int_{-i \infty+0}^{i \infty+0} \frac{d \xi}{\xi} Z_{i}(r, \xi, 0) B_{i}(\xi) \\
& \times\left[(I-r s A(-\xi))^{-1} A(-\xi)\right]_{i k} .
\end{aligned}
$$

Let $U_{k}(s, n)=E\left(s C_{n} 1\left(Y_{n}=k\right)\right)$ and $U(s, n)$ the $N$-dimensional column vector with components $U_{k}(s, n)$, then

$$
\begin{aligned}
\sum_{n=1}^{\infty} r^{n} U(s, n)= & r\left(\Lambda-Q^{T}\right)\left((1-r s) \Lambda-Q^{T}\right)^{-1} p \\
& +r(1-s) \frac{1}{2 \pi i} \int_{-i \infty+0}^{i \infty+0} \frac{d \xi}{\xi} \Lambda\left(-\xi I+(1-r s) \Lambda-Q^{T}\right)^{-1} \\
& \times B(\xi) Z(r, \xi, 0) .
\end{aligned}
$$


The process $\left(\left(C_{n}, Y_{n}\right), n=1,2, \ldots\right)$ is regenerative with the same regeneration points as the process $\left(\left(W_{n}, Y_{n}\right), n=1,2, \ldots\right)$, because the events $\left\{C_{n}=0\right\}$ and $\left\{W_{n}=0\right\}$ are identical. Therefore $\left(C_{n}, Y_{n}\right)$ converges weakly to a stationary vector $(C, Y)$ iff $\rho<1$. For $\rho<1$ let $U_{k}(s)=E\left(s^{C} 1(Y=k)\right)$ and $U(s)$ the column vector with components $U_{k}(s)$. Then

$$
U(s)=(1-s) \frac{1}{2 \pi i} \int_{-i \infty+0}^{i \infty+0} \frac{d \xi}{\xi} \Lambda\left(-\xi I+(1-s) \Lambda-Q^{T}\right)^{-1} B(\xi) Z(\xi) .
$$

As $\left\{C_{n}=0\right\}$ and $\left\{W_{n}=0\right\}$ are identical we have

$$
U(0)=Z_{0}, \text { where } U_{k}(0)=P(C=0, Y=k) .
$$

This also follows from (4.24) by using (4.21).

Since we have assumed that there exists a $\delta>0$ such that $B(\phi)$ can be continued analytically to the region $\operatorname{Re} \phi>-\delta$, we see from (4.3) that the same applies to $Z(\phi)$. Expression (4.17) implies

$$
Z(0)=\lambda^{-1} \Lambda Z^{*}(0)=\lambda^{-1} \Lambda \pi
$$

so that

$$
\begin{aligned}
(1- & s) \Lambda\left((1-s) \Lambda-Q^{T}\right)^{-1} Z(0) \\
= & \lambda^{-1} \Lambda\left((1-s) \Lambda-Q^{T}\right)^{-1}(1-s) \Lambda \pi \\
= & \lambda^{-1} \Lambda \pi+\lambda^{-1} \Lambda\left((1-s) \Lambda-Q^{T}\right)^{-1} Q^{T} \pi=\lambda^{-1} \Lambda \pi
\end{aligned}
$$

Consequently from (4.24) we have for $|s|<1$,

$$
U(s)=\lambda^{-1} \Lambda \pi+\frac{1}{2 \pi i} \int_{-i \infty-0}^{i \infty-0} \frac{d \xi}{\xi}(1-s) \Lambda\left(-\xi I+(1-s) \Lambda-Q^{T}\right)^{-1} B(\xi) Z(\xi) .
$$

We easily see that

$$
\begin{aligned}
& \frac{\partial^{n}}{\partial s^{n}}(1-s) \Lambda\left(-\xi I+(1-s) \Lambda-Q^{T}\right)^{-1} \\
& \quad=n !\left[\Lambda\left(-\xi I+(1-s) \Lambda-Q^{T}\right)^{-1}\right]^{n+1}\left(\xi I+Q^{T}\right) \Lambda^{-1} .
\end{aligned}
$$

The integral in (4.26) converges uniformly in $s$ for $0<s<1$, so that the first derivative of $U(s)$ is found by differentiating the integrand with respect to $s$. Similarly we find the higher derivatives so that for the nth derivative of $U(s)$ we obtain from (4.26) and (4.27),

$$
\begin{aligned}
U^{(n)}(s)= & \frac{n !}{2 \pi i} \int_{-i \infty-0}^{i \infty-0} \frac{d \xi}{\xi}\left[\Lambda\left(-\xi I+(1-s) \Lambda-Q^{T}\right)^{-1}\right]^{n+1} \\
& \cdot\left(\xi I+Q^{T}\right) \Lambda^{-1} B(\xi) Z(\xi), \text { and } \\
U^{(n)}(1)= & \frac{(-1)^{n+1} n !}{2 \pi i} \int_{-i \infty-0}^{i \infty-0} \frac{d \xi}{\xi}\left[\Lambda\left(\xi I+Q^{T}\right)^{-1}\right]^{n} B(\xi) Z(\xi) .
\end{aligned}
$$

Let $\tau_{1}, \tau_{2}, \ldots, \tau_{N}$ be the eigenvalues of $-Q^{r}$ with $\tau_{1}=0$ and $\operatorname{Re} \tau_{i}>0, i=2, \ldots, N$. Assume that they are distinct. The right eigenvector of $-Q^{T}$ corresponding with $\tau_{\text {, }}$ is 
denoted by $V^{j}$ and $V$ is the $N \times N$-matrix the $j$ th column of which is $V^{j}$. Let $V_{j}^{-1}$ be the $j$ th row of $V^{-1}$. Note that we can take $V^{1}=\pi$ and $V_{1}^{-1}=1$, where 1 is the $N$-dimensional row vector $(1,1, \ldots, 1)$. With these notations we may write

$$
\left(\xi I+Q^{T}\right)^{-1}=V \operatorname{diag}\left(\frac{1}{\xi}, \frac{1}{\xi-\tau_{2}}, \ldots, \frac{1}{\xi-\tau_{N}}\right) V^{-1} .
$$

Inserting (4.30) into (4.29) for $n=1$ and applying contour integration yields

$$
\begin{gathered}
U^{\prime}(1)=\frac{1}{2 \pi i} \int_{-i \infty 0-0}^{i \infty-0} \frac{d \xi}{\xi^{2}} \Lambda \pi 1 B(\xi) Z(\xi) \\
+\sum_{j=2}^{N} \frac{1}{2 \pi i} \int_{-i \infty-0}^{i \infty 0-0} \frac{d \xi}{\xi} \Lambda V^{j} V_{j}^{-1} \frac{1}{\xi-\tau_{j}} B(\xi) Z(\xi) \\
=-\Lambda \pi 1\left[B^{\prime}(0) Z(0)+B(0) Z^{\prime}(0)\right] \\
\\
+\sum_{j=2}^{N} \Lambda V^{j} V_{j}^{-1} \tau_{j}^{-1}\left[B(0) Z(0)-B\left(\tau_{j}\right) Z\left(\tau_{j}\right)\right], \quad \text { or } \\
U^{\prime}(1)=\Lambda \pi[\rho / \lambda+E W]+\sum_{j=2}^{N} \bar{V}_{j} \tau_{j}^{-1}\left[\lambda^{-1} \Lambda \pi-B\left(\tau_{j}\right) Z\left(\tau_{j}\right)\right], \quad \text { where } \\
\bar{V}_{j}=\Lambda V^{j} V_{j}^{-1} .
\end{gathered}
$$

In the same way we may find explicit expressions for $U^{(n)}(1), n=2,3, \ldots$; from (4.29) and (4.30).

For $U^{\prime \prime}(1)$ we find

$$
\begin{aligned}
U^{\prime \prime}(1)= & \Lambda \pi\left[\sum_{i=1}^{N} \pi_{i} \lambda_{i} \beta_{i}^{(2)}+2 \lambda \sum_{i=1}^{N} \beta_{i} E(W 1(Y=i))+\lambda E W^{2}\right] \\
& +2 V_{i}^{*}\left[Z^{\prime}(0)-\lambda^{-1} \beta \Lambda \pi\right] \\
& +2\left[\left(\sum_{j=2}^{N} \bar{V}_{j} \tau_{j}^{-1}\right)^{2}-\sum_{j=2}^{N}\left(\bar{V}_{1} \bar{V}_{j}+\bar{V}_{j} \bar{V}_{1}\right) \tau_{j}^{-2}\right] \lambda^{-1} \Lambda \pi \\
& +2 \sum_{j=2}^{N} \bar{V}_{j}^{2} \tau_{j}^{-1}\left[B^{\prime}\left(\tau_{j}\right) Z\left(\tau_{j}\right)+B\left(\tau_{j}\right) Z^{\prime}\left(\tau_{j}\right)\right] \\
& +2 \sum_{j=2}^{N}\left[V_{j}^{*} \tau_{j}^{-1}-\bar{V}_{j}^{2} \tau_{j}^{-2}\right] B\left(\tau_{j}\right) Z\left(\tau_{j}\right),
\end{aligned}
$$

where $\beta_{i}^{(2)}=B_{i}^{\prime \prime}(0)$ and

$$
V_{j}^{*}=\sum_{\substack{k=1 \\ k \neq j}}^{N}\left(\bar{V}_{j} \bar{V}_{k}+\bar{V}_{k} \bar{V}_{j}\right) \frac{1}{\tau_{j}-\tau_{k}}, \quad j=1,2, \ldots, N .
$$

The vectors $Z\left(\tau_{j}\right)$ and $Z^{\prime}\left(\tau_{j}\right)$ can be solved from (4.3). For the first two moments of $C$ we now have

$$
E(C 1(Y=k))=U_{k}^{\prime}(1) \quad E\left(C^{2} 1(Y=k)\right)=U_{k}^{\prime \prime}(1)+U_{k}^{\prime}(1) .
$$

4.4. Number of customers in the system in continuous time. The number of custom- 
ers in the system at time $t$ is denoted by $C_{t}^{*}$. From the relation

$$
\begin{aligned}
\left\{C_{t}^{*}<j\right\} & =\left\{N_{t}<j\right\} \cup \bigcup_{n=1}^{\infty}\left\{T_{n}+W_{n}+S_{n}<t, N_{t}=n+j\right\} \\
& =\left\{t<T_{j+1}\right\} \cup \bigcup_{n=1}^{\infty}\left\{T_{n}+W_{n}+S_{n}<t, T_{n+j}<t<T_{n+j+1}\right\}
\end{aligned}
$$

we find analogous to the derivation of (4.23), for $\operatorname{Re} \eta>\operatorname{Re} \xi,|s|<1$,

$$
\begin{aligned}
\int_{0}^{\infty} \exp (-\eta t) U^{*}(s, t) d t= & s\left(\eta I+(1-s) \Lambda-Q^{T}\right)^{-1} p \\
& +(1-s) \frac{1}{2 \pi i} \int_{-i \infty+0}^{i \infty+0} \frac{d \xi}{\xi}\left((\eta-\xi) I+(1-s) \Lambda-Q^{T}\right)^{-1} \\
& \times B(\xi) Z(1, \xi, \eta)
\end{aligned}
$$

where $U^{*}(s, t)$ is the $N$-dimensional column vector with components

$$
U_{k}^{*}(s, t)=E\left(s^{C_{t}^{*}} 1\left(X_{t}=k\right)\right) \text {. }
$$

The process $\left(\left(C_{t}^{*}, X_{t}\right), t>0\right)$ is regenerative with the same regeneration epochs as $\left(\left(W_{t}^{*}, X_{t}\right), t>0\right)$. Consequently $\left(C_{t}^{*}, X_{t}\right)$ converges for $t \rightarrow \infty$ to a stationary vector $\left(C^{*}, X\right)$ iff $\rho<1$. For $\rho<1$ let $U_{k}^{*}(s)=E\left(s^{*} 1(X=k)\right)$ and $U^{*}(s)$ the column vector with components $U_{k}^{*}(s)$. With (4.16) we find from (4.35) for $|s|<1$,

$$
U^{*}(s)=(1-s) \frac{1}{2 \pi i} \int_{-i \infty+0}^{i \infty+0} \frac{d \xi}{\xi}\left(-\xi I+(1-s) \Lambda-Q^{T}\right)^{-1} B(\xi) \lambda Z(\xi) .
$$

By comparing (4.36) with (4.24) we see that

$$
U^{*}(s)=\lambda \Lambda^{-1} U(s)
$$

The probabilities $P\left(C^{*}=0, X=j\right)$ and the moments $E\left(C^{* n} 1(X=j)\right)$ are now easily expressed in the corresponding quantities for $(C, Y)$.

5. Numerical algorithm. The analysis in the previous sections implies a numerical algorithm for the computation of several quantities of interest. If $\rho<1$ this algorithm yields exact results for the steady state distributions of:

-the actual waiting time $W$;

- the virtual waiting time $W^{*}$;

-the total number of customers in the system at arrival epochs $C$;

- the total number of customers in the system in continuous time $C^{*}$.

In particular we can calculate the probabilities that these random variables are equal to zero as well as all their moments. We shall restrict ourselves to the first two moments.

In this section we give a summary of some important definitions and results, a description of the numerical algorithm and some numerical examples.

For a complete description of the model the following quantities have to be specified:

$N$ : the number of states of the underlying Markov chain $\left(X_{t}, t>0\right)$;

$Q$ : the generator of this Markov chain;

$\Lambda=\operatorname{diag}\left(\lambda_{1}, \lambda_{2}, \ldots, \lambda_{N}\right)$, where $\lambda_{i}$ is the arrival rate while the underlying Markov chain is in state $i$;

$B(\phi)=\operatorname{diag}\left(B_{1}(\phi), B_{2}(\phi), \ldots, B_{N}(\phi)\right)$, where $B_{j}(\phi)$ is the Laplace-Stielties transform 
of the service time of a customer arriving while the underlying Markov chain is in state $i$.

Moreover we denote

$$
\begin{aligned}
\beta & =\operatorname{diag}\left(\beta_{1}, \beta_{2}, \ldots, \beta_{N}\right), \\
\beta^{(k)} & =\operatorname{diag}\left(\beta_{1}^{(k)}, \beta_{2}^{(k)}, \ldots, \beta_{N}^{(k)}\right), \quad k=2,3, \ldots ;
\end{aligned}
$$

where $\beta_{i}$ is the first moment and $\beta_{i}^{(k)}$ the $k$ th moment corresponding to $B_{i}(\phi)$, i.e. $\beta_{i}=-B_{i}^{\prime}(0)$ and $\beta_{i}^{(k)}=(-1)^{k} B_{i}^{(k)}(0)$.

We have defined for $i=1,2, \ldots, N$;

$$
\begin{array}{cc}
Z_{i}(\phi)=E(\exp (-\phi W) 1(Y=i)), & Z_{i}^{*}(\phi)=E\left(\exp \left(-\phi W^{*}\right) 1(X=i)\right), \\
U_{i}(s)=E\left(s^{c} 1(Y=i)\right), & U_{i}^{*}(s)=E\left(s^{c^{*}} 1(X=i)\right),
\end{array}
$$

and $Z(\phi), Z^{*}(\phi), U(s)$ and $U^{*}(s)$ as the column vectors the $i$ th components of which are $Z_{i}(\Phi), Z_{i}^{*}(\Phi), U_{i}(s)$ and $U_{i}^{*}(s)$ respectively. Furthermore we denote the stationary distribution of the Markov chain $\left(X_{t}, t>0\right)$ by the $N$-dimensional column vector $\pi$, the mean arrival rate by $\lambda=\sum_{i=1}^{N} \pi_{i} \lambda_{i}$ and the traffic intensity by $\rho=\sum_{i=1}^{N} \pi_{i} \lambda_{i} \beta_{i}$.

From (4.17) and (4.37) we have for $i=1,2, \ldots, N$ :

$$
Z_{i}^{*}(\phi)=\left(\lambda / \lambda_{i}\right) Z_{i}(\phi), \quad U_{i}^{*}(\phi)=\left(\lambda / \lambda_{i}\right) U_{i}(\phi) .
$$

This implies the intuitive fact that

$$
\begin{gathered}
E\left(\exp \left(-\phi W^{*}\right) \mid X=i\right)=E(\exp (-\phi W) \mid Y=i) \\
E\left(s^{c^{*}} \mid X=i\right)=E\left(s^{c} \mid Y=i\right)
\end{gathered}
$$

For a convenient presentation of the numerical results we introduce the following additional notations:

the stationary distribution of the Markov chain $\left(Y_{n}, n=1,2, \ldots\right)$ is denoted by the $N$-dimensional column vector $\gamma$; from (5.1) we have $\gamma_{i}=\pi_{i} \lambda_{i} / \lambda, i=1,2, \ldots, N$;

the mean expected service time $\beta=\sum_{i=1}^{N} \gamma_{i} \beta_{i}$;

the coefficient of variation $c_{i}^{2}=\beta_{i}^{(2)} / \beta_{i}^{2}-1$;

$P 0_{i}=P(W=0 \mid Y=i)=P(C=0 \mid Y=i)=P\left(W^{*}=0 \mid X=i\right)=P\left(C^{*}=0 \mid X\right.$

$=i)$ :

$E W_{i}=E(W \mid Y=i)=E\left(W^{*} \mid X=i\right)$

$E W_{i}^{2}=E\left(W^{2} \mid Y=i\right)=E\left(W^{* 2} \mid X=i\right)$

$\operatorname{var} W_{i}=E W_{i}^{2}-\left(E W_{i}\right)^{2}$

$E C_{i}=E(C \mid Y=i)=E\left(C^{*} \mid X=i\right)$

$E C_{i}^{2}=E\left(C^{2} \mid Y=i\right)=E\left(C^{* 2} \mid X=i\right)$

$\operatorname{var} C_{i}=E C_{i}^{2}-\left(E C_{i}\right)^{2}$

Next we give a stepwise description of the numerical algorithm:

1. Compute $\pi$ from the system $Q^{T_{\pi}}=0$ and the normalizing condition $1 \pi=1$; compute $\lambda=1 \Lambda \pi, \beta=1 \beta \gamma$ and $\rho=\lambda \beta$.

2. Determine the zeros $\mu_{1}=0, \mu_{2}, \ldots, \mu_{N}$ in the right half-plane of

$$
\operatorname{det} L(\phi)=\operatorname{det}\left(-\phi I+(I-B(\phi)) \Lambda-Q^{T}\right),
$$

and the corresponding nonzero row vectors $F_{1}, F_{2}, \ldots, F_{N}$ satisfying $F_{i} L\left(\mu_{i}\right)=0$, $i=1,2, \ldots, N$. Compute the row vectors

$$
D_{i}=F_{i}\left(-\mu_{i} I+\Lambda-Q^{r}\right), \quad i=1,2, \ldots, N
$$

and let $D$ be the $N \times N$-matrix the $i$ th row of which is $D_{i}$. 
3. Determine the eigenvalues $\nu_{1}, \nu_{2}, \ldots, \nu_{N}$ (in the right half-plane) of $\Lambda-Q^{T}$ and the corresponding right eigenvectors $R^{1}, R^{2}, \ldots, R^{N}$; and let $R$ be the $N \times N$-matrix the $i$ th column of which is $R^{i}$.

4. Compute the matrix $S$ with $S_{i j}=D_{i} \Lambda R^{j} /\left(\nu_{j}-\mu_{i}\right), i=1,2, \ldots, N ; j=1$, $2, \ldots, N$; and the matrix $C=\Lambda R S^{-1}$.

Then for $i=1,2, \ldots, N$;

$$
P 0_{i}=C_{i 1} D_{11}(1-\rho) /\left(\pi_{i} \lambda_{i}\right) .
$$

5. Solve $Z^{\prime}(0)$ and $Z^{\prime \prime}(0)$ from (4.5) then for $i=1, \ldots, N$,

$$
E W_{i}=-Z_{i}^{\prime}(0) / \gamma_{i} ; \quad E W_{i}^{2}=Z_{i}^{\prime \prime}(0) / \gamma_{i} .
$$

6. Determine the eigenvalues $\tau_{1}=0, \tau_{2}, \ldots, \tau_{N}$ (in the right half-plane) of $-Q^{T}$ and the corresponding right eigenvectors $V^{1}=\pi, V^{2}, \ldots, V^{N}$; and let $V$ be the $N \times N$-matrix the $i$ th column of which is $V^{i}$.

7. For $j=1,2, \ldots, N$, solve $Z\left(\tau_{j}\right)$ and $Z^{\prime}\left(\tau_{j}\right)$ from (4.3). Compute $U^{\prime}(1)$ and $U^{\prime \prime}(1)$ using (4.32) and (4.33) then we have for $i=1,2, \ldots N$,

$$
E C_{i}=U_{i}^{\prime}(1) / \gamma_{i} ; \quad E C_{i}^{2}=\left[U_{i}^{\prime \prime}(1)+U_{i}^{\prime}(1)\right] / \gamma_{i} .
$$

The algorithm can be used whenever the following conditions are satisfied:

$-\lambda_{i}>0, i=1,2, \ldots, N$

$-\tau_{1}, \tau_{2}, \ldots, \tau_{N}$ are distinct;

$-\mu_{1}, \mu_{2}, \ldots, \mu_{N}, \nu_{1}, \nu_{2}, \ldots, \nu_{N}$ are all distinct;

$-\operatorname{det} S \neq 0$.

In the examples that we have considered we have chosen $\Lambda$ and $Q$ such that the first two conditions were satisfied. It turned out that for these choices the last two conditions were also satisfied.

It is seen that any model for which the conditions are not all satisfied can be approximated arbitrarily closely by a model for which they are. We have considered examples for which $\tau_{1}, \tau_{2}, \ldots, \tau_{N}$ are all real and examples for which some of them are not. In the first case $\mu_{1}, \mu_{2}, \ldots, \mu_{N}$ and $\nu_{1}, p_{2}, \ldots, \nu_{N}$ turned out to be also real. Our numerical experience has led us to the following conjectures.

Conjecture 5.1. If $\lambda_{i}>0, i=1,2, \ldots, N$, and $\tau_{1}, \tau_{2}, \ldots, \tau_{N}$ are distinct then $\mu_{1}, \mu_{2}, \ldots, \mu_{N}, \nu_{1}, \nu_{2}, \ldots, \nu_{N}$ are all distinct and $\operatorname{det} S \neq 0$.

Conjecture 5.2. If $\tau_{1}, \tau_{2}, \ldots, \tau_{N}$ are all real then so are $\mu_{1}, \mu_{2}, \ldots, \mu_{N}, \nu_{1}$, $\nu_{2}, \ldots, v_{N}$.

We have not been able to prove these conjectures. If $\tau_{1}, \tau_{2}, \ldots, \tau_{N}$ are all real the $\mu_{1}, \mu_{2}, \ldots, \mu_{N}$ are easily calculated using an improved regula falsi. In the case that some of the $\tau_{i}$ are not real we have restricted ourselves to rational $B(\phi)$ so that $\mu_{1}, \mu_{2}, \ldots, \mu_{N}$ are zeros of a polynomial and can be calculated using Bairstow's method.

ExAMPLE 5.1 (Burman and Smith [2], Model 2 with exponential holding times). $N=6, \rho=0.8, \lambda=0.8, \beta=1$.

\begin{tabular}{rcccc|rrrrrr}
\hline$i$ & $\lambda_{i}$ & $G_{i}$ & $\beta_{i}$ & $c_{i}^{2}$ & $j$ & 1 & \multicolumn{7}{c}{$Q_{i j}$} & \multicolumn{1}{c}{3} & \multicolumn{1}{c}{4} & \multicolumn{1}{c}{5} & \multicolumn{1}{c}{6} \\
\hline 1 & 0.00 & $M$ & 1.00 & 1.00 & -0.5 & 0.5 & 0.0 & 0.0 & 0.0 & 0.0 \\
2 & 0.32 & $M$ & 1.00 & 1.00 & 0.1 & -0.5 & 0.4 & 0.0 & 0.0 & 0.0 \\
3 & 0.64 & $M$ & 1.00 & 1.00 & 0.0 & 0.2 & -0.5 & 0.3 & 0.0 & 0.0 \\
4 & 0.96 & $M$ & 1.00 & 1.00 & 0.0 & 0.0 & 0.3 & -0.5 & 0.2 & 0.0 \\
5 & 1.28 & $M$ & 1.00 & 1.00 & 0.0 & 0.0 & 0.0 & 0.4 & -0.5 & 0.1 \\
6 & 1.60 & $M$ & 1.00 & 1.00 & 0.0 & 0.0 & 0.0 & 0.0 & 0.5 & -0.5 \\
\hline
\end{tabular}




\begin{tabular}{cccccccc}
\hline$i$ & $\pi_{i}$ & $\gamma_{i}$ & $P 0_{i}$ & $E W_{i}$ & $\operatorname{var} W_{i}$ & $E C_{i}$ & $\operatorname{var} C_{i}$ \\
\hline 1 & 0.03125 & 0.00000 & 0.52063 & 3.6569 & 47.632 & 3.6569 & 43.975 \\
2 & 0.15625 & 0.06250 & 0.34840 & 4.6157 & 54.328 & 4.6157 & 49.712 \\
3 & 0.31250 & 0.25000 & 0.22507 & 5.6844 & 61.071 & 5.6844 & 55.386 \\
4 & 0.31250 & 0.37500 & 0.14082 & 6.8466 & 67.716 & 6.8466 & 60.869 \\
5 & 0.15625 & 0.25000 & 0.08558 & 8.0859 & 74.163 & 8.0859 & 66.077 \\
6 & 0.03125 & 0.06250 & 0.05065 & 9.3872 & 80.355 & 9.3872 & 70.967 \\
\hline
\end{tabular}

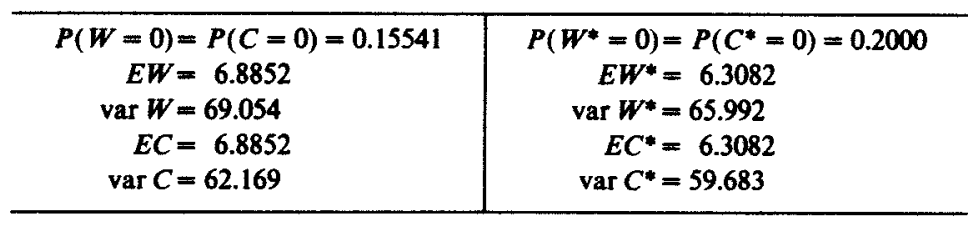

ExAMPLe 5.2 (Burman and Smith [2], Model 2 with constant holding times). $N=6$, $\rho=0.8, \lambda=0.8, \beta=1$.

\begin{tabular}{rrrrr|rrrrrrr}
\hline$i$ & $\lambda_{i}$ & $G_{i}$ & $B_{i}$ & $c_{t}^{2}$ & $j$ & 1 & 2 & 3 & 4 & 5 & 6 \\
\hline 1 & 0.00 & $D$ & 1.00 & 0.00 & -0.5 & 0.5 & 0.0 & 0.0 & 0.0 & 0.0 \\
2 & 0.32 & $D$ & 1.00 & 0.00 & 0.1 & -0.5 & 0.4 & 0.0 & 0.0 & 0.0 \\
3 & 0.64 & $D$ & 1.00 & 0.00 & 0.0 & 0.2 & -0.5 & 0.3 & 0.0 & 0.0 \\
4 & 0.96 & $D$ & 1.00 & 0.00 & 0.0 & 0.0 & 0.3 & -0.5 & 0.2 & 0.0 \\
5 & 1.28 & $D$ & 1.00 & 0.00 & 0.0 & 0.0 & 0.0 & 0.4 & -0.5 & 0.1 \\
6 & 1.60 & $D$ & 1.00 & 0.00 & 0.0 & 0.0 & 0.0 & 0.0 & 0.5 & -0.5 \\
\hline
\end{tabular}

\begin{tabular}{cccccccc}
\hline$i$ & $\pi_{i}$ & $\gamma_{i}$ & $P 0_{i}$ & $E W_{i}$ & $\operatorname{var} W_{i}$ & $E C_{i}$ & var $C_{i}$ \\
\hline 1 & 0.03125 & 0.00000 & 0.62504 & 1.8316 & 17.335 & 2.0289 & 18.539 \\
2 & 0.15625 & 0.06250 & 0.38863 & 2.5815 & 21.352 & 2.8907 & 22.428 \\
3 & 0.31250 & 0.25000 & 0.22526 & 3.4974 & 25.593 & 3.8846 & 26.466 \\
4 & 0.31250 & 0.37500 & 0.12291 & 4.5572 & 29.857 & 4.9941 & 30.511 \\
5 & 0.15625 & 0.25000 & 0.06370 & 5.7322 & 33.988 & 6.1987 & 34.452 \\
6 & 0.03125 & 0.06250 & 0.03161 & 6.9954 & 37.900 & 7.4783 & 38.219 \\
\hline
\end{tabular}

\begin{tabular}{c|c}
$P(W=0)=P(C=0)=0.14460$ & $P\left(W^{*} x=0\right)=P\left(C^{*}=0\right)=0.20000$ \\
$E W=4.6149$ & $E W^{*}=4.0919$ \\
$\operatorname{var} W=31.033$ & $\operatorname{var} W^{*}=29.079$ \\
$E C=5.0417$ & $E C^{*}=4.4919$ \\
$\operatorname{var} C=31.792$ & $\operatorname{var} C^{*}=29.985$ \\
\hline
\end{tabular}

Example 5.3 (Van Hoorn and Seelen [5, Table 1] with $\rho=0.6, \theta=0.2$ ). $\quad N=2$, $\rho=0.6, \lambda=0.6, \beta=1$.

\begin{tabular}{rrrrr|rrr}
\hline$i$ & & & & & \multicolumn{2}{c}{$Q_{i j}$} \\
\hline 1 & $\lambda_{i}$ & $G_{i}$ & $\beta_{i}$ & $c_{i}^{2}$ & $j$ & \multicolumn{1}{c}{1} \\
\hline 2 & 0.92399 & $H_{2}$ & 1 & 4 & & -0.0446 & 0.0446 \\
\hline
\end{tabular}

\begin{tabular}{cccccccc}
\hline$i$ & $\pi_{i}$ & $\gamma_{i}$ & $P O_{i}$ & $E W_{i}$ & var $W_{i}$ & $E C_{i}$ & var $C_{i}$ \\
\hline 1 & 0.56880 & 0.87594 & 0.23754 & 6.9817 & 101.443 & 5.8880 & 61.741 \\
2 & 0.43120 & 0.12406 & 0.61430 & 3.3583 & 59.084 & 2.7138 & 36.651 \\
\hline
\end{tabular}




\begin{tabular}{c|c}
\hline$P(W=0)=P(C=0)=0.28428$ & $P\left(W^{*}=0\right)=P\left(C^{*}=0\right)=0.40000$ \\
$E W=6.5321$ & $E W^{*}=5.4193$ \\
$\operatorname{var} W=97.614$ & $\operatorname{var} W^{*}=86.398$ \\
$E C=5.4942$ & $E C^{*}=4.5193$ \\
$\operatorname{var} C=59.723$ & $\operatorname{var} C^{*}=53.394$ \\
\hline
\end{tabular}

EXAMPLE 5.4. A case in which the eigenvalues of $-Q^{T}$ are real. $N=8, \rho=0.9$, $\lambda=0.9, \beta=1$.

\begin{tabular}{|c|c|c|c|c|c|c|c|c|c|c|c|c|}
\hline \multirow[b]{2}{*}{$i$} & \multirow[b]{2}{*}{$\lambda_{1}$} & \multirow[b]{2}{*}{$G_{i}$} & \multirow[b]{2}{*}{$\beta_{i}$} & \multirow[b]{2}{*}{$c_{i}^{2}$} & \multicolumn{8}{|c|}{$Q_{i j}$} \\
\hline & & & & & $j$ & 2 & 3 & 4 & 5 & 6 & 7 & 8 \\
\hline 1 & 0.41976 & $\boldsymbol{M}$ & 0.41253 & 1.0000 & -12 & 6 & 2 & 1 & 1 & 0 & 1 & 1 \\
\hline 2 & 0.83952 & $D$ & 1.03132 & 0.0000 & 3 & -8 & 2 & 2 & 1 & 0 & 0 & 0 \\
\hline 3 & 1.34323 & $E_{2}$ & 1.23758 & 0.5000 & 1 & 4 & -9 & 3 & 0 & 1 & 0 & 0 \\
\hline 4 & 1.13335 & $\mathrm{H}_{2}$ & 1.15250 & 1.3533 & 0 & 0 & 3 & -5 & 2 & 0 & 0 & 0 \\
\hline 5 & 0.62964 & $D$ & 0.59301 & 0.0000 & 0 & 1 & 0 & 2 & -7 & 3 & 1 & 0 \\
\hline 6 & 0.16790 & $E_{2}$ & 0.92819 & 0.5000 & 0 & 0 & 0 & 1 & 3 & -6 & 1 & 1 \\
\hline 7 & 1.00742 & $M$ & 1.46963 & 1.0000 & 1 & 1 & 1 & 2 & 1 & 2 & -10 & 2 \\
\hline 8 & 1.67904 & $E_{3}$ & 0.54144 & 0.3333 & 0 & 0 & 0 & 0 & 1 & 1 & 2 & -4 \\
\hline
\end{tabular}

\begin{tabular}{cccccccc}
\hline$i$ & $\pi_{i}$ & $\gamma_{i}$ & $P 0_{i}$ & $E W_{i}$ & var $W_{i}$ & $E C_{i}$ & $\operatorname{var} C_{i}$ \\
\hline 1 & 0.04734 & 0.02208 & 0.09988 & 9.3642 & 110.64 & 9.3399 & 100.46 \\
2 & 0.12903 & 0.12036 & 0.09670 & 9.4119 & 110.71 & 9.3693 & 100.49 \\
3 & 0.13060 & 0.19492 & 0.09225 & 9.5092 & 111.07 & 9.4282 & 100.58 \\
4 & 0.25741 & 0.32415 & 0.09653 & 9.4452 & 111.04 & 9.3896 & 100.56 \\
5 & 0.17540 & 0.12271 & 0.10533 & 9.2581 & 110.41 & 9.2895 & 100.44 \\
6 & 0.13820 & 0.02578 & 0.11379 & 9.1813 & 110.22 & 9.2109 & 100.36 \\
7 & 0.05041 & 0.05643 & 0.10213 & 9.3044 & 110.56 & 9.3325 & 100.53 \\
8 & 0.07160 & 0.13357 & 0.09146 & 9.2550 & 110.16 & 9.4645 & 100.74 \\
\hline
\end{tabular}

\begin{tabular}{c|c}
$P(W=0)=P(C=0)=0.09695$ & $P\left(W^{*}=0\right)=P\left(C^{*}=0\right)=0.10000$ \\
$E W=9.3888$ & $E W^{*}=9.3554$ \\
$\operatorname{var} W=110.762$ & $\operatorname{var} W^{*}=110.683$ \\
$E C=9.3835$ & $E C^{*}=9.3499$ \\
$\operatorname{var} C=100.558$ & $\operatorname{var} C^{*}=100.517$ \\
\hline
\end{tabular}

EXAMPLE 5.5. A case in which some eigenvalues of $-Q^{r}$ are nonreal. $N=5$, $\rho=0.95, \lambda=0.95, \beta=1$.

\begin{tabular}{rcccc|rrrrrr}
\hline$i$ & $\lambda_{i}$ & $G_{i}$ & $\beta_{i}$ & $c_{i}^{2}$ & $j$ & 1 & 2 & 3 & 4 & 5 \\
\hline 1 & 0.65242 & $M$ & 1.27265 & 1.0000 & -4 & 1 & 1 & 1 & 1 \\
2 & 0.97863 & $E_{2}$ & 1.07380 & 0.5000 & 1 & -5 & 2 & 0 & 2 \\
3 & 0.32621 & $H_{2}$ & 0.17101 & 1.0757 & 3 & 3 & -9 & 1 & 2 \\
4 & 1.30484 & $E_{3}$ & 2.14760 & 0.3333 & 1 & 2 & 1 & -8 & 4 \\
5 & 1.63105 & $M$ & 0.63633 & 1.0000 & 3 & 2 & 1 & 0 & -6 \\
\hline
\end{tabular}

\begin{tabular}{cccccccc}
\hline$i$ & $\pi_{i}$ & $\gamma_{i}$ & $P 0_{i}$ & $E W_{i}$ & $\operatorname{var} W_{i}$ & $E C_{i}$ & var $C_{i}$ \\
\hline 1 & 0.33906 & 0.23285 & 0.05202 & 19.7130 & 432.73 & 19.6788 & 412.15 \\
2 & 0.25521 & 0.26290 & 0.04906 & 19.7556 & 432.70 & 19.7315 & 412.18 \\
3 & 0.12552 & 0.04310 & 0.05319 & 19.6556 & 432.54 & 19.6619 & 412.14 \\
4 & 0.05807 & 0.07976 & 0.04935 & 19.9290 & 433.49 & 19.7195 & 412.15 \\
5 & 0.22214 & 0.38138 & 0.04635 & 19.7702 & 432.74 & 19.8150 & 412.33 \\
\hline
\end{tabular}




\begin{tabular}{c|c}
$P(W=0)=P(C=0)=0.04892$ & $P\left(W^{*}=0\right)=P\left(C^{*}=0\right)=0.05000$ \\
$E W=19.7608$ & $E W^{*}=19.7419$ \\
$\operatorname{var} W=432.78$ & $\operatorname{var} W^{*}=432.75$ \\
$E C=19.7471$ & $E C^{*}=19.7227$ \\
$\operatorname{var} C=412.23$ & $\operatorname{var} C^{*}=412.20$ \\
\hline
\end{tabular}

The algorithm requires that all $\lambda_{i}$ be strictly positive. Therefore in Example 5.1 we have chosen $\lambda_{1}=10^{-12}$. In Examples 5.4 and 5.5 the $q_{i}$ are relatively large compared to the $\lambda_{i}$, i.e. the Markov chain is "fast" compared to the arrival process. This explains why $P 0_{i}, E W_{i}$, var $W_{i}, E C_{i}$ and $\operatorname{var} C_{i}$ do not vary much for different values of $i$. In Example 5.3 the Markov chain is relatively slow whereas in Examples 5.1 and 5.2 the $q_{i}$ and the $\lambda_{i}$ are of the same order of magnitude. The computer time needed for the calculations is very modest. For each of the Examples 5.4 and 5.5 the required CPU-time on a DEC 2060 was about 10 seconds.

\section{References}

[1] Arjas, E. (1972). On the Use of a Fundamental Identity in the Theory of Semi-Markov Queues. Adv. in Appl. Probab. 4 271-284.

[2] Burman, D. Y. and Smith, D. R. (1983). An Asymptotic Analysis of a Queueing System with Markov-Modulated Arrivals. Bell Laboratories, Holmdel, N.J.

[3] de Smit, J. H. A. (1983). The Queue GI|M|s with Customers of Different Types or the Queue $G I\left|H_{m}\right| s$. Adv. in Appl. Probab. 15 392-419.

[4] Ramaswami, V. (1980). The N|G|1 Queue and Its Detailed Analysis. Adv. in Appl. Probab. 12 222-261.

[5] van Hoom, M. H. and Seelen, L. P. (1983). The SPP|G|1 Queue: A Single Server Queue with a Switched Poisson Process as Input Process. O. R. Spektrum 5 207-218.

DEPARTMENT OF APPLIED MATHEMATICS, TWENTE UNIVERSITY OF TECHNOLOGY, P.O. BOX 217, N-7500 AE ENSCHEDE, THE NETHERLANDS 